\title{
Analysis of Capture and Multi-Packet Reception on the AIS Satellite System
}

\author{
Federico Clazzer, Andrea Munari
}

\begin{abstract}
In this paper, we discuss the impact of the capture effect and multi-user detection on the reception of automatic identification system messages at the satellite. Resorting to simple yet beneficial tools such as the multi-packet reception matrix and the random access approximation of the medium access layer, an insightful model is presented. A comparison with the destructive collision model is also carried out and performance evaluation with vessel distribution and vessel speed distribution gathered from real data are derived. The more realistic physical layer improves the results accuracy and provides tradeoffs and future research directions.
\end{abstract}

\section{INTRODUCTION}

In the last years, satellite-aided automatic identification system (AIS) [1] has become one of the hot topics in the maritime safety and security field, drawing the attention of both researchers and standardization bodies [2]. The possibility to monitor vessel routes worldwide opens the road to an unexplored plurality of commercial applications. Goods and ship tracking as well as oceans monitoring for both environmental and safety purposes are only some of the most relevant scenarios.

Although the reception of AIS packets at the satellite is viable and already demonstrated [3], [4], [5], [6], the standard has been originally developed for inter-vessel radio communications. In particular, the AIS self-organized time division multiple access (SOTDMA) medium access (MAC) protocol [1] is designed in a way that most of packet collisions can be avoided among vessels that are in the reciprocal radio reception range. ${ }^{1}$ While this approach has been proven to be robust for ship-to-ship and ship-to-shore communications, its effectiveness may degrade significantly when a satellite attempts to collect AIS traffic. In fact, the satellite field of view is orders of magnitude greater than the vessels radio reception range and thus prone to destructive collisions among packets of different ships. Especially in densely vessel populated regions

Federico Clazzer is with the Institute of Communication and Navigation of the Deutsches Zentrum für Luft- und Raumfahrt (DLR), D-82234 Wessling, Germany (e-mail: federico.clazzer@dlr.de). Andrea Munari is with the Institute for Networked Systems of the RWTH Aachen University, D-52072 Aachen, Germany (e-mail: andrea.munari@inets.rwth-aachen.de). This work has been accepted for publication in IEEE/MTS Oceans'15, Genova. (C)2015 IEEE. Personal use of this material is permitted. Permission from IEEE must be obtained for all other uses, in any current or future media, including reprinting /republishing this material for advertising or promotional purposes, creating new collective works, for resale or redistribution to servers or lists, or reuse of any copyrighted component of this work in other works.

${ }^{1}$ SOTDMA is in fact the the most used out of four access schemes foreseen in AIS. A vessel implementing it broadcasts its position, velocity and direction along with other data. In order to distribute up-to-date information to neighbouring vessels, messages are sent more often as the ship speed increases, resulting in 4 possible frequencies of packet transmission. For more details on SOTDMA, refer to [1], [7]. this issue affects dramatically the tracking performance of a satellite-aided AIS system, all the more so considering the steadily increasing traffic generated by other maritime communication services being allocated to VHF band [8].

The design of more advanced reception schemes has thus become a primary concern. In this perspective, the possibility of reliably predicting the performance of satellite-AIS and of identifying the key tradeoffs that impact the system is paramount. While some results are available in the literature, they either are mainly based on simulations and measurement campaigns (and thus intrinsically depend on the specific configurations being tested) [3], [4], [5] or study in details one aspect of the AIS system [9]. On the other hand, a unified framework capable of accounting for topological, MAC and physical (PHY) layer issues and flexible enough to support different system configurations to gather a clearer understanding of the main performance drivers is still missing. A first step in this direction was taken by the work in [7], showing that the MAC protocol of AIS as perceived at a satellite can be well approximated resorting to random access such as slotted ALOHA (SA). This result is particularly interesting, as it allows to abstract the non-trivial details of SOTDMA and characterise the performance at the MAC layer through a single channel traffic coefficient. An analytical expression of such parameter was derived in [10] for any flying object capable of receiving AIS data, thus including satellites. The channel traffic is then expressed as a function of the vessels number in the satellite reception range as well as their speed. Nevertheless, these works rely on a simplified physical layer, the so-called destructive collision channel model, assuming that all packets involved in a collision are lost, while messages received over a collision-free slot are decoded with probability 1. Despite its mathematical tractability, this abstraction is not always realistic and might not be detailed enough. Conversely, an interesting analytical characterisation of different and more accurate PHY layer models for random access (RA) schemes was derived in [11], [12] but the results are instantiated for terrestrial scenarios and overlook some specific and peculiar aspects of AIS, e.g., the topological distribution of ships or the communications system parameters.

Taking the lead from this, in the present work we will extend the analysis of AIS at the satellite, assuming a realistic physical layer and introducing both capture and multi-packet reception effects. The physical layer of AIS will take into account the specific modulation scheme adopted and a realistic link budget will be carried out. From these elements, a simple yet effective interference model will be derived which can then be exploited for deriving the performance of AIS reception at the satellite. A remarkable outcome will be the assessment 
of the satellite reception capabilities of AIS messages with advanced receivers that may apply multi-user detection (MUD) techniques as, for example, successive interference cancellation (SIC). On the other hand, the MAC layer will exploit the model of SOTDMA as SA derived in [7].

The reminder of the paper is organized as follows. In Section II the analytical framework is presented, including both MAC and PHY layers. The latter is then discussed for three possible scenarios and the multi-packet reception (MPR) matrix representation is introduced. In Section III, the MPR matrices for the three scenarios are instantiated along with a realistic AIS link budget. Numerical results in terms of throughput and first pass detection probability follow in Section IV. Finally, a summary on the key findings and conclusion are closing the paper in Section V.

\section{An Analytical Framework FOR AIS PERFORMANCE}

In order to offer a compact yet insightful characterisation of the performance of satellite-aided AIS, we introduce in this section an analytical framework that identifies the key performance drivers of the system while being flexible enough to support different models for both MAC and PHY layers. Throughout our work, we focus on a satellite in low Earth orbit (LEO) at latitude and longitude coordinates $\left(\varphi_{s}, \lambda_{s}\right)$, whose footprint covers a region $\mathcal{A}$ on the Earth surface. All vessels equipped with an AIS device within such region generate traffic in the form of packets following the SOTDMA protocol [1] over frames of $N_{s}$ slots. Transmitters are assumed to be slot-synchronous, and data units sent over the same slot reach the LEO receiver aligned in time. ${ }^{2}$

A precise description of the resulting traffic profile perceived at the satellite is in general non-trivial, as not only does it depend on ship density and speed distribution, but also on the dynamic composition of AIS radio clusters among vessels. On the other hand, the broad LEO footprint allows collection of packets transmitted by a large number of clusters that are too far apart to coordinate, inducing collision patterns at the flying receiver that tightly match the ones of a random access scheme. Taking the lead from this observation, [7] introduced a simplified yet accurate traffic model for satelliteAIS, abstracting the SOTDMA protocol by considering a population of vessels that access the wireless channel in a fully uncoordinated fashion as per SA random access protocol. An excellent performance approximation was in particular obtained describing the number of packets $U$ sent over a slot by ships within $\mathcal{A}$ as a Poisson random variable of parameter $G$, referred to as channel load (or channel traffic), so that:

$$
\operatorname{Pr}\{U=u\}=\frac{e^{-G} G^{u}}{u !} .
$$

The approach of [7] represents a relevant modelling result, as it effectively embeds a large set of protocol details into a single parameter that suffices to compute the overall

\footnotetext{
${ }^{2}$ For the sake of mathematical tractability, we do not consider misalignment in time at the receiver due to different propagation delays undergone by packets transmitted from different positions within the footprint. Similarly, no Doppler effect is considered.
}

performance. From this standpoint, notice that any technique providing an estimate of the average channel load can in fact be plugged into the presented framework to gather MAC layer statistics. Among the possible choices, we will refer in the present work to the solution proposed in [10], and express

$$
G=\sum_{i} \frac{\omega_{i}}{N_{s}}\left(n \int_{V_{i-1}}^{V_{i}} f_{v}(v) \mathrm{d} v\right) \quad[\mathrm{pk} / \mathrm{slot}]
$$

Here, $\omega_{i}$ is the number of messages per frame a ship is mandated to send per AIS when travelling at velocity $v \in$ $\left[V_{i-1}, V_{i}\right]$. Thus, defining $f_{v}$ as the probability density function (PDF) of vessel speed, the summation in (2) evaluates the average number of packets per slot generated in $\mathcal{A}$ by properly weighting the fractions of the $n$ transmitters seen at the satellite that fall within each velocity range. In turn, the vessel population seen at the satellite can be computed by integrating the ship density $f_{s}$ over $\mathcal{A}$ :

$$
n=\int_{\mathcal{A}} f_{s}(\varphi, \lambda) \mathrm{d} \varphi \mathrm{d} \lambda
$$

In order to estimate the channel load, then, the approach of Eqs. (2)-(3) simply requires the PDF of ship position and speed, which can be derived via dedicated models, simulations or, as will be done throughout our work, extracted from realworld collected data.

The characterisation of the traffic profile offered by the discussed MAC abstraction represents a first and key step towards a model of the overall performance. In this perspective, in fact, a satellite-aided AIS system is typically evaluated in terms of either the aggregate throughput $\mathcal{T}$, defined as the average number of messages successfully decoded per slot, or the first pass detection probability $\zeta$, indicating the probability that a ship is discovered (i.e., decoded at least once) during the time a satellite flying by covers its location. ${ }^{3}$ While both metrics are certainly influenced by the channel load, they also depend on the probability of decoding a message, and are thus intrinsically related to the physical layer as well as to the receiver capabilities. In this way, an accurate model of the physical layer brings the advantage of improving the precision of the performance results. In bringing these aspects into the analytical framework, we consider three alternative scenarios:

- destructive collision model (CO): in this case, a message is correctly decoded as long as it is the only one transmitted over a slot, whereas the superposition of two or more packets at the satellite prevents retrieval of any of them. The collision channel is a common reference for the study of random access protocols. Moreover, by virtue of its high level of abstraction, the $\mathrm{CO}$ leads to compact and insightful closed form expressions for most of the metrics of interest at the MAC level, e.g., $\mathcal{T}_{\text {co }}=G e^{-G}$. The next sections will shed light on the accuracy of such a handy approximation, discussing in which conditions it can be reliably used to capture the overall performance of satellite-aided AIS.

\footnotetext{
${ }^{3}$ The first pass detection probability is particularly relevant in practical systems, since subsequent fly-bys of the satellite over the same area may occur hours or even days later depending on the orbit.
} 
- capture model (CA): The main drawback of the CO scenario is its abrupt characterisation of the outcome of collisions. In order to further investigate the impact of interference, we introduce a more accurate model which describes the wireless channel connecting a vessel to the satellite as subject to path loss and fading. For the latter, we adopt a Rayleigh model, capturing the large amount of reflections the signal transmitted by an omni-directional AIS antenna typically undergo on the metallic surfaces of a ship. Accordingly, a message reaches the receiver with a power which is exponentially distributed with average $\nu$ and probability density function $f_{P}(a)=(1 / \nu) e^{-a / \nu}$, $a \geq 0$. The mean value follows $\nu \propto P_{t x} d^{-2}$, where $P_{t x}$ is the transmitted power and $d$ is the distance between the sender and the receiver. Packets transmitted over the same slot can thus reach the satellite with different power levels, allowing the receiver to synchronise to the strongest signal. In this case, the packet is said to be captured, and decoding can be attempted regarding all other overlapping messages as additional noise. While the specific probability of successfully retrieving the strongest packet out of a collision depends on the underlying PHY layer and decoder, the capture effect has been shown to trigger remarkable gains in random access settings [11], [12].

- successive interference cancellation model (IC): The performance of satellite-aided AIS can be further boosted when more advanced decoders capable of MPR are considered. This line of research has recently attracted relevant attention also in practical systems [9], as it enables the possibility to extract more than one message out of a collision. Among the different implementations of MPR, we focus on SIC, and consider once more signals affected by path loss and Rayleigh fading. As for the CA, in case of a collision the decoder synchronises to the message with highest power and attempts decoding. In case of success, the receiver subtracts the retrieved packet from the original waveform and attempts decoding on the second strongest message, which now sees an overall level of interference reduced by the contribution of the removed information unit. The procedure is repeated until either the decoding of the packets is successful or up to a maximum number of iterations imposed by decoder complexity or delay constraints. ${ }^{4}$

A flexible modelling tool to comprehensively discuss the three PHY scenarios of interest is offered by the MPR matrix, first introduced in [14]. Considering a collision size $u$ at the receiver, i.e., a slot where $u$ messages are concurrently transmitted, let us define as $\alpha_{u, c}$ the probability of successfully retrieving $c$ packets. Assuming that at most $m$ data units can be decoded, e.g., due to complexity constraints at the receiver,

\footnotetext{
${ }^{4}$ Throughout our work we consider perfect SIC, where the interference contribution of a decoded packet is completely removed. For further details on the accuracy of this hypothesis as well as on SIC in general, the reader is referred to [13]
}

the $m$-column MPR matrix $\boldsymbol{A}_{m}$ is defined as

$$
\boldsymbol{A}_{m}=\left(\begin{array}{cccc}
\alpha_{1,0} & \alpha_{1,1} & 0 & 0 \\
\alpha_{2,0} & \alpha_{2,1} & \alpha_{2,2} & 0 \\
\vdots & \vdots & \vdots & \vdots \\
\alpha_{m, 0} & \alpha_{m, 1} & \ldots & \alpha_{m, m} \\
\alpha_{m+1,0} & \alpha_{m+1,1} & \ldots & \alpha_{m+1, m} \\
\vdots & \vdots & \vdots & \vdots
\end{array}\right)
$$

Taking advantage of this notation, the average throughput can be derived in a compact form for any underlying PHY as

$$
\mathcal{T}=\boldsymbol{\pi} \boldsymbol{A}_{m} \boldsymbol{v}_{m}
$$

where $\pi$ is a row vector whose $u$-th element expresses the probability for $u$ users to transmit over the slot of interest (expressed, e.g., by (1) under the Poisson traffic model), while $\boldsymbol{v}_{m}=[12 \ldots m]^{T}$. $^{5}$

Equation (5) is particularly useful, as it isolates the effects of MAC and PHY layer: the former is captured by $\pi$, which describes how vessels access the medium, whereas $\boldsymbol{A}_{m}$ expresses the latter quantifying the capability of handling collisions. Two final remarks are in order. First, we stress that any MAC policy can be plugged into the model as long as the $\pi$ vector it induces is available, and that decoding schemes other than the ones considered here can be taken into account. Secondly, to the aim of the present discussion the form of the MPR matrix can promptly be instantiated for the three models of interest. In fact, when the $\mathrm{CO}$ model is employed, $\boldsymbol{A}_{m}$ becomes the binary 2-column matrix

$$
\boldsymbol{A}^{(\mathrm{co})}=\left(\begin{array}{cc}
0 & 1 \\
1 & 0 \\
1 & 0 \\
\vdots & \vdots
\end{array}\right)
$$

as decoding occurs only for a collision size one. Similarly, when the capture effect comes into play, a two-column MPR can be written in the form

$$
\boldsymbol{A}^{\text {(ca) }}=\left(\begin{array}{cc}
\alpha_{1,0}^{(\mathrm{ca})} & \alpha_{1,1}^{(\mathrm{ca})} \\
\alpha_{2,0}^{(\mathrm{ca})} & \alpha_{2,1}^{\text {(ca) }} \\
\vdots & \vdots
\end{array}\right)
$$

since at most one packet can be retrieved regardless of the collision size. Incidentally, let us also remark that in contrast to the $\mathrm{CO}$ model, decoding may fail even in the absence of interference (i.e., $\alpha_{1,1}^{(\mathrm{ca})}<1$ ) due to channel impairments. Finally, for the IC case the expression of the MPR matrix takes the general form of (4). The ultimate performance achievable when resorting to these PHY layers clearly depends on the

$$
\begin{aligned}
& { }^{5} \text { The product among matrices with infinite rows in (5) can readily be } \\
& \text { expanded obtaining (in the case of Poissonian traffic): } \\
& \qquad \mathcal{T}=\sum_{u=0}^{\infty} \frac{e^{-G} G^{u}}{u !}\left(\sum_{c=0}^{\min \{u, m\}} c \alpha_{u, c}\right)
\end{aligned}
$$


specific configuration of the communications parameters for the AIS system. In the following section, we will then instantiate the MPR matrices considering realistic values, later used to collect numerical results.

\section{THE MPR MATRIX FOR SATELLITE AIS}

To instantiate the MPR matrices discussed in Section II to the satellite-AIS, we start by observing that their elements depend on the signal-to-interference and noise ratio (SINR) distribution as well as on the PHY characteristic, i.e. modulation and coding. The former, in turn, is the result of several effects, which can be categorized in topological- and linkrelated ones.

As far as topology is concerned, the distribution of ships over earth (i.e., the PDF $f_{s}$ employed in (3)), as well as the actual size of the footprint illuminated by the satellite when at coordinates $\left(\varphi_{s}, \lambda_{s}\right)$ define the path loss AIS transmitters experience to the receiver. We derive the vessel distribution from data collected by the operating satellite-AIS system of exactEarth ${ }^{\circledR}$. In particular, we base our results on the density map reported in Fig. 1, which shows the PDF of the vessels positions coming from data collected in January 2013. The satellite footprint is instead derived considering the very high frequency (VHF) antenna pattern of a LEO satellite flying at $524 \mathrm{~km}$ altitude, for a radius of $2500 \mathrm{~km}$.

The VHF link budget for each vessel-satellite pair is computed based on the next two equations. The mean $\nu$ of the received power is:

$$
\nu=\frac{P_{t x} G_{t x} G_{r x}}{L}
$$

where $G_{t x}$ and $G_{r x}$ are the transmitter and receiver antenna gains respectively, while $L$ is the free space loss depending on the vessel-satellite distance $d$ and on the link frequency $\phi$. The antenna gains for both the transmitter and receiver are conservatively chosen to be $0 \mathrm{~dB}$ [15] and constant. The transmitting power is fixed to $12.5 \mathrm{~W}$ as the class-A AIS transceivers characteristics prescribes [1]. No other atmospheric impairment is taken into account, given the margin for possible additional losses introduced with the conservative assumption on the antenna gains. The received signal-to-noise ratio $(\mathrm{SNR}), \gamma$ is then,

$$
\gamma=\frac{P_{r x}}{T_{a} k B},
$$

where $P_{r x}$ is the received power (exponentially distributed with mean value $\nu$ ), $T_{a}$ is the antenna noise temperature while $B$ is the bandwidth. The former is assumed to be $50 \mathrm{~K}$, while the latter follows the AIS standard and equals $25 \mathrm{kHz}$. The summary of link budget parameters can be found in Table I.

Once the SINR distribution is defined, the elements of the MPR matrices can be evaluated by means of the packet error rate (PER) vs. SINR curve, which fully describes PHY effects. Since coding is not present in the AIS system, we compute the PER curve based on the bit error rate (BER) performance of gaussian minimum shift keying (GMSK) modulation, following the analytical approximation derived in [16]. The PER $P_{p}$ is $P_{p}=1-\left(1-P_{b}\right)^{N_{b}}$, where $P_{b}$ is the BER and $N_{b}$ is

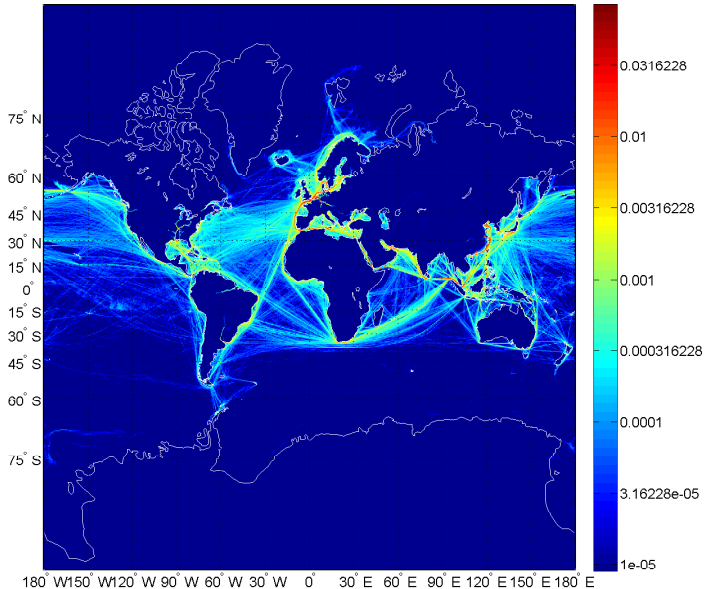

Fig. 1. PDF of vessel positions [\%] derived from exactEarth ${ }^{\circledR}$ data collected in January 2013. The PDF has been computed based on the received AIS messages from the satellite system of exactEarth ${ }^{\circledR}$.

TABLE I

PARAMETERS OF THE VHF LINK BUDGET OD SATELLITE-AIS

\begin{tabular}{ccl}
\hline Parameter & Value & Description \\
\hline$P_{t x}$ & $12.5 \mathrm{~W}$ & Transmitting power \\
$P_{r x}$ & computed & Receiving power \\
$G_{t x}$ & $0 \mathrm{~dB}$ & Transmitting antenna gain \\
$G_{r x}$ & $0 \mathrm{~dB}$ & Receiving antenna gain \\
$\phi$ & $162 \mathrm{MHz}$ & Link frequency \\
$d$ & computed & Vessel-satellite distance \\
$c$ & $3 \cdot 10^{8} \mathrm{~m} / \mathrm{s}$ & Speed of light constant \\
$L$ & $\left(\frac{4 \pi d \phi}{c}\right)^{2}$ & Free space loss \\
$T_{a}$ & $50 \mathrm{~K}$ & Antenna noise temperature \\
$B$ & $25 \mathrm{kHz}$ & Carrier bandwidth \\
$k$ & $1.379 \cdot 10^{-23} \mathrm{~J} / \mathrm{K}$ & Boltzmann constant \\
\hline
\end{tabular}

the number of bits composing a received packet. In case of collision, we model the interfering power following the wellknown Gaussian approximation.

While the presented model offers a very accurate estimate of the decoding probabilities, the SINR distribution shall be derived separately for any satellite position, i.e., $f_{s}=f_{s}\left(\varphi_{s}, \lambda_{s}\right)$. This may often be computationally burdensome, as it would require the numerical computation of the elements of the MPR matrices for any possible collision size at all locations. In order to overcome this issue, we considered the use of a simpler uniform distribution of vessels over the reception area $\mathcal{A}$. Following this approach, the SINR only depends on the size of the illuminated footprint, but not on the specific ship density seen in the region. To validate the assumption, numerical simulations were performed, computing the MPR matrices for any satellite position using both the uniform and the realistic distribution based on exactEarth ${ }^{\circledR}$ data. Results showed that the difference is relatively low, not exceeding $20 \%$ in throughput for the operating conditions of interest ( $G<4 \mathrm{pk} / \mathrm{slots}$ ). Due to these reasons, throughout the paper we will consider the uniform distribution approximation for the derivation of the MPR matrices, abstracting the PHY 


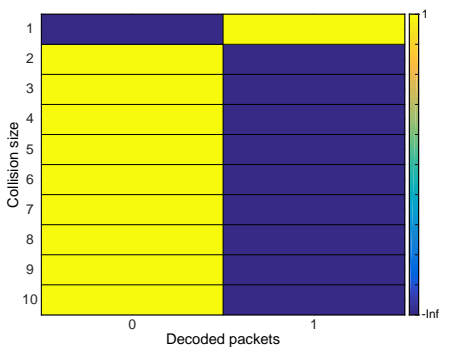

(a) CO MPR matrix.

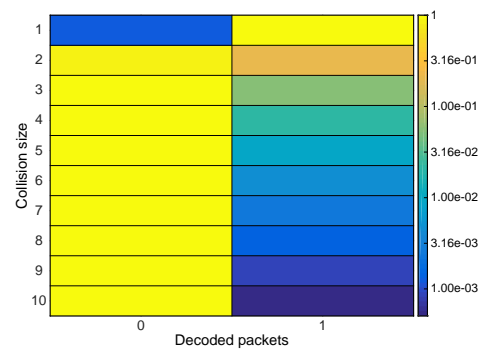

(b) CA MPR matrix of the satellite AIS system considering Rayleigh fading.

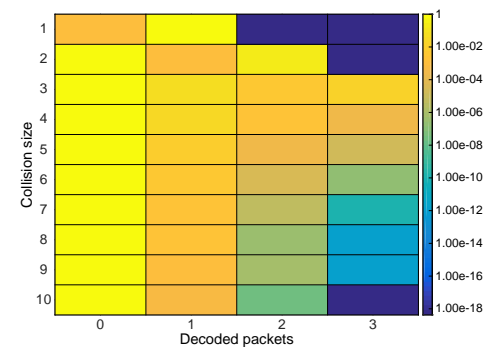

(c) IC MPR matrix of the satellite AIS system considering Rayleigh fading and SIC.

Fig. 2. MPR matrices under $\mathrm{CO}, \mathrm{CA}$ and IC PHY model assumption.

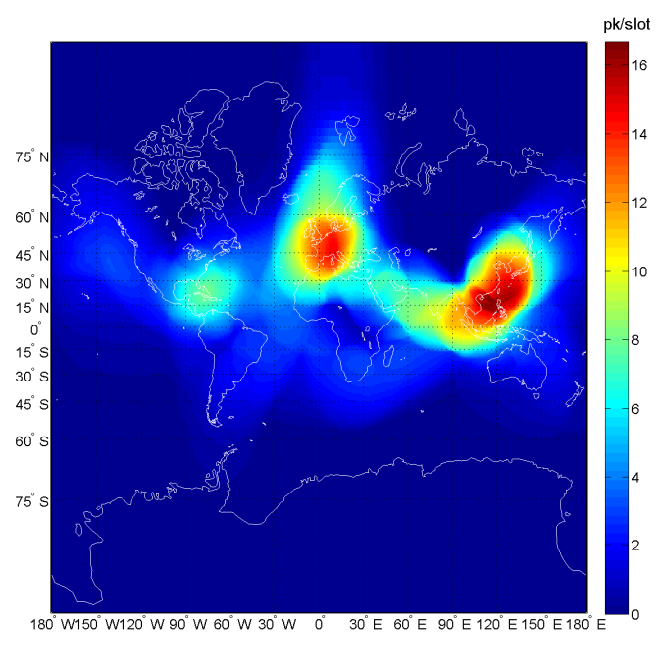

Fig. 3. Global average channel load $G$ measured in pk/slot, observed by a LEO satellite with a coverage radius of $2500 \mathrm{~km}$.

models as independent of the satellite position. Nevertheless, the impact of realistic vessel routes still plays a key role as performance driver and will be thoroughly taken into account for the estimation of the channel load $G$ (see Section IV).

In Fig. 2 the MPR matrix entries for the three PHY models of Section II are depicted. Under CO, a packet can be successfully decoded with probability 1 only if it has been received in a slot without suffering any collision (see also $\boldsymbol{A}^{(\mathrm{co})}$ in (6)). On the other hand, whenever a collision happens, all the packets involved are lost. In this way, the only possible entries for the MPR matrix under the $\mathrm{CO}$ are either 1 or 0, as shown in Fig. 2(a). When the collision size, i.e. the number of packets received in a slot, is equal to 1 the probability of decoding a packet is 1 (and the probability of not decoding is 0 ). For all the other collision sizes, the situation is mirrored, being 1 the probability of not decoding a received packet and 0 the corresponding successful decoding probability. When both capture effect and Rayleigh fading are considered, as in the CA MPR matrix shown in Fig. 2(b), the probability of decoding a packet also in presence of a collision is non-zero (see also the mathematical expression of $\boldsymbol{A}^{\text {(ca) }}$ in (7)). For small collision sizes - up to 3 colliding packets - the probability of decoding one packet is higher than $10^{-2}$. Increasing the collision size reduces the probability of correctly decoding one packet, which becomes vanishing for a collision size around 10 . At the same time, also when the packet is received collision-free, the successful decoding probability is lower than 1 . Nevertheless, due to the very high transmitting power and narrow carrier adopted in AIS, this probability is still very close to 1 . Enabling SIC at the receiver allows to correctly decode more than one packet per slot, as shown in Fig. 2(c). In particular, in case of 2 colliding packets, the probability of decoding both of them is close to $2 \cdot 10^{-2}$, while the probability of decoding only one is much lower, $10^{-3}$. The reason lies in the fact that, once we are able to correctly decode one packet, SIC removes entirely its interference contribution from the slot, leaving the second packet collision-free. Since AIS has a very high transmitting power and narrow carrier, the probability of correctly decoding a packet collision-free is very close to one also in presence of Rayleigh fading, as seen for the CA case, inducing a high probability of decoding both packets. Remarkably, in case of a collision size of 3 , the probability of successfully decoding all the received packets is still $1.5 \cdot 10^{-2}$. As in the previous case, as long as the collision size increases, the probabilities of decoding one or more packets fall very close to 0 . In the following section, we will exploit these MPR matrices for showing the performance of satellite-AIS in terms of different metrics, i.e. throughput and first pass detection probability.

\section{Performance Evaluation}

In order to evaluate the system performance of interest, the channel load $G$ experienced at the satellite has to be estimated. As shown in (2)-(3), $G$ depends on the satellite position $\left(\varphi_{s}, \lambda_{s}\right)$ and reception radius, on the ship density $f_{s}$ and on the vessel speed PDF $f_{v}$. For the LEO system under study, the PDF of the ship density is computed based on satellite AIS data collected in January 2013 and shown in Fig. 1. We are assuming a total worldwide number of vessels with active AIS transceiver equal to $65 \cdot 10^{3}$. The vessel speed PDF is also computed based on exactEarth ${ }^{\circledR}$ data of the same month and year.

The resulting global average channel load for such a LEO satellite is shown in Fig. 3. Three regions show the highest channel load, the Gulf of Mexico, the region covering the central part of the Mediterranean Sea and the English Strait, 
and the Chinese coast. The first region shows channel loads as high as $8 \mathrm{pk} / \mathrm{slot}$, while the second and third regions can reach channel loads exceeding $14 \mathrm{pk} /$ slot. Furthermore, along the most adopted routes (Singapore, Red Sea and Mediterranean Sea) channel loads as high as $6 \mathrm{pk} / \mathrm{slot}$ can be found.

\section{A. Throughput}

The throughput performance of (5) is shown in Fig. 4 for the three PHY models. In all cases, the throughput map can be divided into three regions. The first one, that covers the Antarctica continent in the southern hemisphere and the Arctic region in the northern hemisphere excluding the Svalbard, exhibits a very limited throughput. In this region in fact, the number of vessels is particularly low and therefore channel load seen at the satellite is marginal. The second region is delimited by the Mediterranean Sea, north Africa, central Europe and the center of the Atlantic Ocean. Also in this region the throughput is exiguous but the reason is opposite. The number of vessels seen by the satellite is enormous, generating a very high traffic which results in a collisions that bound the throughput close to 0 . The third and last region connects the first two and there the throughput reaches its maximum. In this belt in fact, the channel load is quite limited, reaching up to $4-5 \mathrm{pk} / \mathrm{slot}$. Although similar in the coverage, the absolute throughput values are different for the three PHY models. When both capture effect and SIC are enabled, throughputs up to $0.46 \mathrm{pk} / \mathrm{slot}$ can be reached, compared to the well-known maximum of $0.36 \mathrm{pk} / \mathrm{slot}$ of the SA model under CO. Furthermore, the belt corresponding to high throughput has a larger width under IC compared to both $\mathrm{CO}$ and CA.

Aiming to give a better overview of the throughput gains reached by the more realistic PHY models compared to the destructive collision channel model, we introduce a specific metric

$$
\xi=\left(\mathcal{T} / \mathcal{T}_{\text {со }}\right)-1
$$

$\xi$ represents the throughput gain of the other two models over the destructive collision channel model, and is shown in Fig. 5 for both CA and IC. The plot reports the values of $\xi$ in $\mathrm{dB}$, so that a 0 value represents a $100 \%$ gain, i.e, twice the throughput w.r.t. the collision channel model. In the maps we have delimited with a black line the region of interest with throughput exceeding $0.25 \mathrm{pk} / \mathrm{slot}$. The throughput gain of CA with respect to the destructive collision channel model, depicted in Fig. 5(a), reaches values up to $20 \mathrm{~dB}$. In the region under discussion, we can see that gains up to $50 \%$ can be achieved when we consider CA. Higher throughput gains can be reached in the very high load regions, which are of less practical impact due to the very limited throughput. The throughput gain of the IC over the $\mathrm{CO}$ is shown in Fig. 5(b). While the maximum throughput gain remains similar to the case of $\mathrm{CA}$, the region with $\mathcal{T}_{\text {ic }} \geq 0.25$ is in this case extended, especially towards the high load region in the center of the global map. Furthermore, the throughput gains in this region are higher reaching up to $70 \%$.

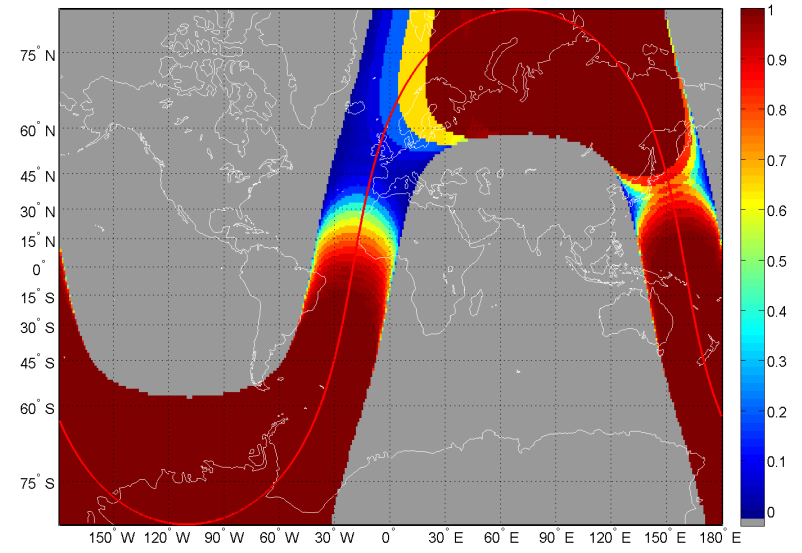

Fig. 6. First pass detection probability $\zeta$ at a LEO satellite with reception radius of $2500 \mathrm{~km}$. The physical layer assumed is the capture effect with SIC model.

\section{B. First Pass Detection Probability}

Although the throughput is a measure of the AIS communication system efficiency, the main aim of satellite-aided AIS is the worldwide tracking of vessels. Following this reasoning, there is a major need for a metric that quantifies the capability of detecting a vessel from a satellite. Following the steps of [10] we define the first pass ship detection probability $\zeta$, as the probability of correctly decoding a packet of any ship located in the specific coordinates illuminated by the flying satellite. Aiming at considering the more realistic channel models discussed in this work, the expression of $\zeta$ in [10] shall be modified as follows

$$
\zeta(\varphi, \lambda, r)=1-\prod_{i=1}^{I(r)}\left(\sum_{u=1}^{\infty} \frac{e^{-G_{i}} G_{i}^{u}}{u !} \alpha_{u, 0}\right)^{k_{i}} .
$$

Here, $k_{i}$ is the average number of AIS messages sent by a ship in the $i$-th frame, $I(r)$ is the number of frames during which the receiver illuminates the location with coordinates $(\varphi, \lambda)$. It is important to note that the channel load $G_{i}(\varphi, \lambda, r)$ depends on the specific location illuminated by the satellite $(\varphi, \lambda)$ as well as by the receiver coverage radius $r$. Finally, $\alpha_{u, 0}$ is simply the probability of not decoding a packet given a collision involving $u$ packets. The first pass detection probability is of interest especially when considering a realistic ground track of a LEO satellite. Due to this, the first pass detection probability $\zeta$ for a possible LEO satellite ground track is shown in Fig. 6, under IC. The vast majority of the ground track show a first pass detection probability equal or very close to 1 , especially when the satellite is passing at high latitudes. Nevertheless, when the satellite is flying over the West part of Europe, the first pass detection probability drops to almost 0 . This is due to the high density region that prevents the ships to be detected due to the large amount of packet collisions. Instead, the probability decrease is less drastic close to the Russian coast in the Pacific Ocean, where the probability is reduced to circa 0.7 . 


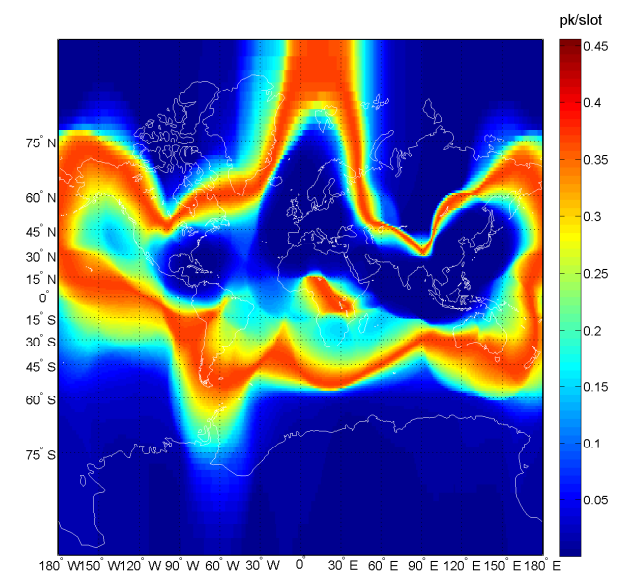

(a) Global average throughput $\mathcal{T}_{\text {co }}$ measured in pk/slot, observed by a LEO satellite with a coverage radius of $2500 \mathrm{~km}$ under CO.

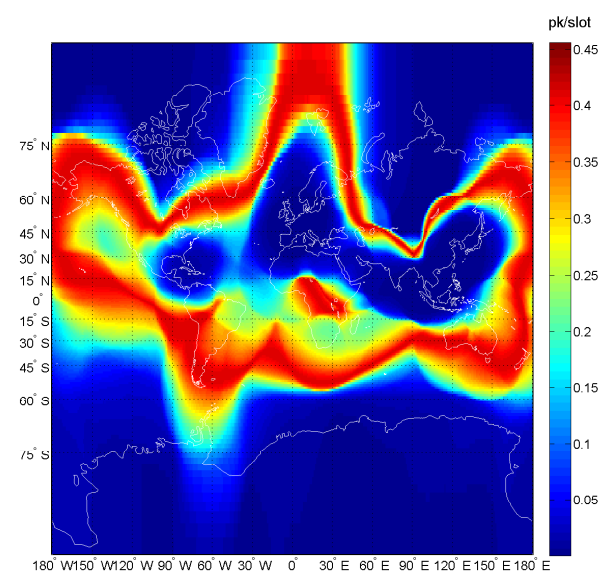

(b) Global average throughput $\mathcal{T}_{\text {ca }}$ measured in pk/slot, observed by a LEO satellite with a coverage radius of $2500 \mathrm{~km}$ under CA.

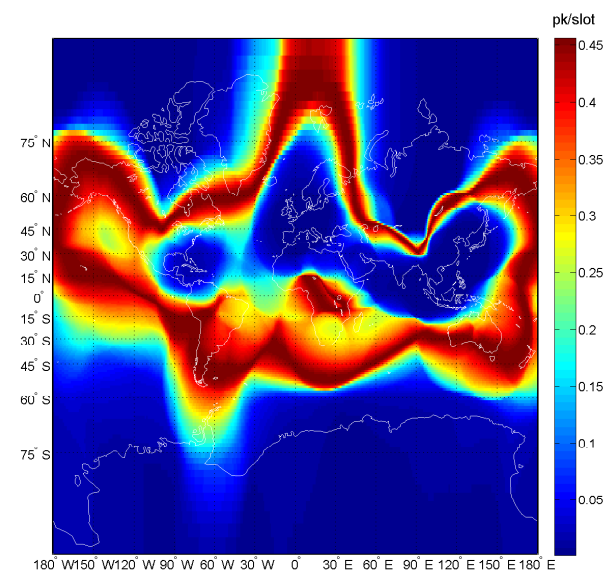

(c) Global average throughput $\mathcal{T}_{\text {ic }}$ measured in pk/slot, observed by a LEO satellite with a coverage radius of $2500 \mathrm{~km}$ under IC.

Fig. 4. Global average throughput $\mathcal{T}$ for the three aforementioned PHY models, CA, CO and IC

The comparison between the three PHY layers is shown in Fig. 7. The first pass detection probability is shown against the satellite longitude, which uniquely determines the satellite position on the ground track of Fig. $6 .^{6}$ The first observation is that the CO approximates very well the more realistic PHY models, i.e. CA and IC. Moreover, depending on the satellite position, the $\mathrm{CO}$ model can be either slightly underestimating or slightly overestimating the first pass detection probability. This is due to the fact, that $\zeta$ on a given location on Earth, depends not only on the channel load when the satellite is located exactly over this specific point, but is a function also of the previous and next satellite positions. Furthermore, due to the specific satellite orbit, different location on Earth are covered by the satellite for different amount of time introducing another level of variability on $\zeta$. Comparing CA and IC, the same first pass detection probability performance

\footnotetext{
${ }^{6}$ Due to the satellite orbit, the longitude intervals between two consecutive satellite positions are not constant over the ground track as it is shown by the $x$-axis labels.
}

can be observed. Here, the gain due to the advanced receiver capability of the IC is less prominent since an average vessel is received several times over a satellite pass. The effectiveness of the receiver improvement on this metric which is concentrating on decoding at least one packet per vessel, is therefore minor.

\section{Summary AND CONCLUSIONS}

In this paper the analysis of satellite-aided AIS, taking into account realistic physical layer effects has been carried out. The propagation phenomena of the AIS VHF link as well as characteristics of both transmitters (vessels) and receiver (satellite) are considered, leading to three PHY layer models. Apart from the destructive collision model (CO), useful for abstracting PHY layer effects and used for reference, two additional models are introduced, the capture model (CA) and the successive interference cancellation model (IC), where vessel-satellite distance and Rayleigh fading are both taken into consideration. The former gives a more realistic behavior of the PHY enabling the correct reception of a packet also in 


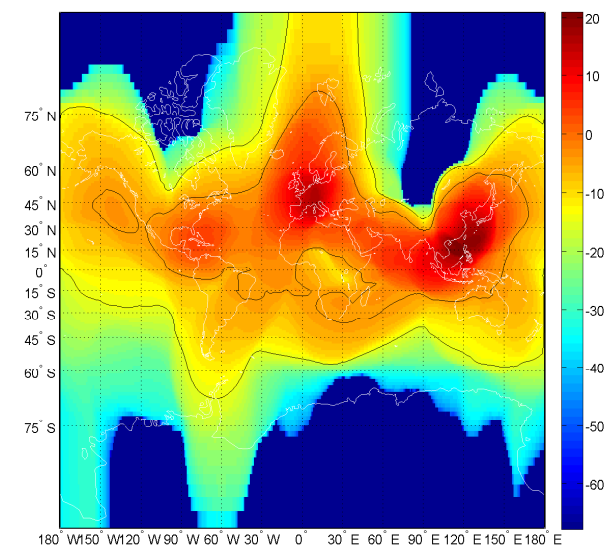

(a) Global average throughput gain $\xi_{d B}$ of $\mathrm{CA}$ with respect to the $\mathrm{CO}$, in dB. The gain follows $\xi=\left(\mathcal{T}_{\mathrm{ca}} / \mathcal{T}_{\mathrm{co}}\right)-1$, and $\xi_{d B}=10 \log _{10}(\xi)$. The LEO satellite has a coverage radius of $2500 \mathrm{~km}$ and a realistic VHF link budget is assumed.

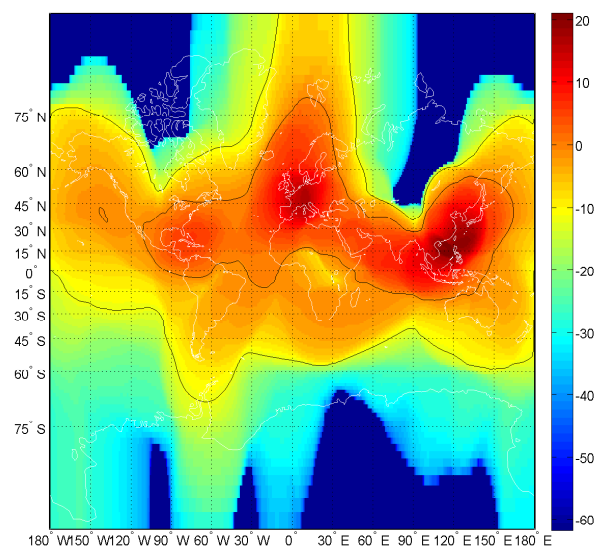

(b) Global average throughput gain $\xi$ of IC with respect to the $\mathrm{CO}$, in $\mathrm{dB}$. The gain follows $\xi=\left(\mathcal{T}_{\text {ic }} / \mathcal{T}_{\text {co }}\right)-1$, and $\xi_{d B}=10 \log _{10}(\xi)$. The LEO satellite has a coverage radius of $2500 \mathrm{~km}$ and a realistic VHF link budget is assumed.

Fig. 5. Global average throughput gain $\xi$ for the three aforementioned PHY models. The black line delimits the throughput region exceeding 0.25 [pk/slot], i.e. the region where $\mathcal{T} \geq 0.25$.

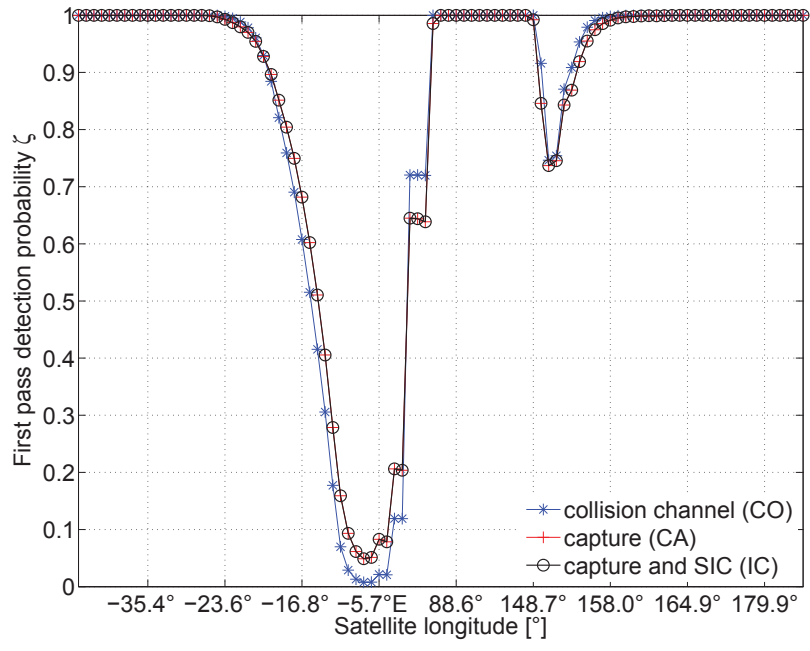

Fig. 7. First pass detection probability $\zeta$ at a LEO satellite with reception radius of $2500 \mathrm{~km}$. $\zeta$ is computed over the ground track of Fig. 6 and compared for the three channel models.

presence of collisions, while the latter improves the receiver performance enabling the capacity of decoding multiple packets exploiting interference cancellation techniques. A common representation of the three models is given thanks to the introduction of the MPR matrix, which is also exploited for giving in a compact form the throughput expression. The satelliteaided AIS system is compared under the three PHY models under both throughput and first pass detection probability. The key results can be summarized as follows:

- the MPR matrices can be well approximated assuming uniform vessel distribution over the satellite reception coverage. This is valid for satellites due to their huge reception range, but might not be valid for other flying receivers such as airliners;

- the throughput performance when considering more realistic PHY models as CA or when the receiver capabilities are improved as with IC, show a remarkable gain w.r.t. the simple $\mathrm{CO}$ model;

- the first pass detection probability estimated with the very compact and simple CO is approximating very well both advanced PHY models.

The tools developed in this work can be exploited for making estimations on satellite-aided AIS system performance with potentially any receiver characteristic, giving deep insights on performance gains from both a communication and system perspectives.

\section{ACKNOWLEDGEMENTS}

The research leading to these results has been carried out under the framework of the project "R\&D for the maritime safety and security and corresponding real time services". The project started in January 2013 and is led by the Program Coordination Defence and Security Research within the German Aerospace Center (DLR).

\section{REFERENCES}

[1] Recommendation ITU-R M.1371-4, "Technical characteristics for an automatic identification ssystem using time-division multiple access in the VHF maritime mobile band," ITU, Tech. Rep., 2010.

[2] Recommendation ITU-R M.2169, "Improved satellite detection of AIS," ITU, Tech. Rep., 2009.

[3] T. Eriksen, G. Hoye, B. Narheim, and J. Meland, "Maritime traffic monitoring using a space-based AIS receiver," Acta Astronautica, vol. 58, pp. 537-549, 2006.

[4] T. Eriksen, A. N. Skauen, B. Narheim, O. Helleren, O. Olsen, and R. B. Olsen, "Tracking Ship Traffic with Space-Based AIS: Experience Gained in First Months of Operations," in Waterside Security Conference (WSS), 2010 International, Carrara, Italy, 3-5 November 2010, pp. 1-8.

[5] J. A. Larsen, H. P. Mortensen, and J. D. Nielsen, "An SDR based AIS Receiver for Satellites," in Recent Advances in Space Technologies (RAST), 2011 5th International Conference on, Istanbul, Turkey, 9-11 June 2011, pp. 526-531. 
[6] J. A. Larsen and H. P. Mortensen, "In Orbit Validation of the AAUSAT3 SDR based AIS receiver," in Recent Advance in Space Technologies (RAST), 2013 6th International Conference on, Istanbul, Turkey, 12-14 June 2013, pp. 487-491.

[7] F. Clazzer, A. Munari, M. Berioli, and F. Lazaro Blasco, "On the Characterization of AIS Traffic at the Satellite," in IEEE/MTS Oceans 14, Taipei, Taiwan, April 2014.

[8] Electronic Communications Committee, "Information Paper on VHF Data Exchange System (VDES), CPG PTC(13) INFO 16," CEPT, Tech. Rep., 2013.

[9] G. Colavolpe, T. Foggi, A. Ugolini, J. Lizarraga, S. Cioni, and A. Ginesi, "A highly efficient receiver for satellite-based automatic identification system signal detection," in Advanced Satellite Multimedia Systems Conference and the 13th Signal Processing for Space Communications Workshop (ASMS/SPSC), Sept 2014, pp. 120-127.

[10] F. Clazzer, A. Munari, S. Plass, and B. Suhr, "On the Impact of Coverage Range on AIS Message Reception at Flying Platforms," in 7th Advanced Satellite Multimedia Systems Conference (ASMS), Livorno, Italy, September 2014, pp. 128-135.
[11] A. Zanella and M. Zorzi, "Theoretical Analysis of the Capture Probability in Wireless Systems with Multiple Packet Reception Capabilities," IEEE Transactions on Communications, vol. 60, no. 4, pp. 1058-1071, April 2012.

[12] A. Munari, F. Clazzer, and G. Liva, "Multi-Receiver Aloha Systems - a Survey and New Results," in IEEE ICC Workshop on Massive Uncoordinated Access Protocols (MASSAP), London, UK, June 2015.

[13] G. Liva, "Graph-based analysis and optimization of contention resolution diversity slotted aloha," Communications, IEEE Transactions on, vol. 59, no. 2, pp. 477-487, february 2011.

[14] S. Ghez, S. Verdú, and S. C. Schwartz, "Stability Properties of Slotted Aloha with Multipacket Reception Capability," IEEE Transactions on Automatic Control, vol. 33, no. 7, pp. 640-649, July 1988.

[15] Report ITU-R M.2084, "Satellite detection of Automatic Identification System Messages,” ITU, Tech. Rep., 2006.

[16] K. Murota and K. Hirade, "GMSK Modulation for Digital Mobile Radio Telephony," IEEE Transactions on Communication, vol. COM-29, no. 7 , pp. 1044-1050, July 1981. 